\title{
Case Report \\ Skin Testing to Identify Safe Drugs for Patients with Rocuronium-Induced Anaphylaxis
}

\author{
Manzo Suzuki (iD, ${ }^{1}$ Hajime Kawase, ${ }^{2}$ Azusa Ogita, ${ }^{3}$ and Hiroyasu Bito $\mathbb{D}^{2}$ \\ ${ }^{1}$ Anesthesiologist, Yatsu Health Hospital, Chiba, Japan \\ ${ }^{2}$ Department of Anesthesiology, Musashikosugi Hospital Nippon Medical School, Kawasaki, Kanagawa, Japan \\ ${ }^{3}$ Department of Dermatology, Musashikosugi Hospital Nippon Medical School, Kawasaki, Kanagawa, Japan
}

Correspondence should be addressed to Manzo Suzuki; manzo@nms.ac.jp

Received 8 October 2019; Accepted 30 December 2019; Published 29 January 2020

Academic Editor: Alparslan Apan

Copyright ( 92020 Manzo Suzuki et al. This is an open access article distributed under the Creative Commons Attribution License, which permits unrestricted use, distribution, and reproduction in any medium, provided the original work is properly cited.

\begin{abstract}
Among patients who develop anaphylaxis during anesthesia, anaphylaxis caused by a neuromuscular blocking agent has the highest incidence. In patients who developed IgE-mediated anaphylaxis, and cross-reactivity among NMBAs is a concern in subsequent anesthetic procedures. We present a patient who developed rocuronium-induced anaphylaxis in whom the skin prick test (SPT) and intradermal test (IDT) could identify a safe drug to use in the subsequent anesthetic procedure. A 32-year-old female developed anaphylactic shock at the induction of general anesthesia. She recovered by administration of hydrocortisone and epinephrine. Skin tests including the SPT followed by the IDT revealed rocuronium as the drug that caused anaphylaxis and vecuronium as a safe drug to use for the subsequent general anesthesia. She safely underwent surgery with general anesthesia using vecuronium one month after the skin testing. There are not many reports on the effectiveness of the SPT followed by IDT in identifying the causative drug as well as a safe drug to use in the subsequent anesthetic procedure following anaphylaxis during anesthesia. The usefulness of the SPT should be re-evaluated.
\end{abstract}

\section{Introduction}

Anaphylaxis during anesthesia is uncommon and is sometimes life-threatening. Neuromuscular blocking agents (NMBAs), latex, and antibiotics are among the causes of anaphylactic reaction during anesthesia [1]. In approximately sixty percent of anaphylaxis cases during anesthesia, anaphylaxis is mediated by an NMBA [2]. Although succinylcholine is associated with a high incidence of anaphylaxis $[3,4]$, the number of reports on anaphylactic and anaphylactoid reaction due to rocuronium has recently been increasing [5].

Administration of an NMBA such as rocuronium can induce an immunoglobulin E- (IgE-) mediated or non-IgEmediated anaphylactic reaction. In patients who developed IgE-mediated anaphylaxis, cross-reactivity among NMBAs is a concern in subsequent anesthetic procedures. The causative drug is identified by testing such as with the skin prick test (SPT) and intradermal test (IDT). In vitro testing, i.e., identification of the specific $\operatorname{IgE}$ against NMBA and basophil activation test (BAT), has become available in recently developed procedures $[6,7]$. In addition to identification of the drug that caused the anaphylaxis reaction, it is important to determine which drug is safe for subsequent anesthetic procedures. We present a patient who developed rocuronium-induced anaphylaxis in whom the anaphylaxis skin test could identify a safe drug to use in the subsequent anesthetic procedure.

\section{Case Presentation}

A 32-year-old female was scheduled to undergo laparoscopic ovarian cystectomy. Her past history was unremarkable except for the presence of contact allergy to metal, and she had never undergone an anesthesia procedure previously. Her height was $164 \mathrm{~cm}$, and weight was $79 \mathrm{~kg}$. An epidural catheter was placed before anesthesia induction. Povidone iodine was used for skin disinfection, and mepivacaine $0.5 \%$ was used for skin infiltration of local anesthetics. During and after placement of the epidural catheter, the patient's 
condition was stable. General anesthesia was induced by propofol $150 \mathrm{mg}$, and continuous infusion of remifentanil $0.3 \mu \mathrm{g} / \mathrm{kg} / \mathrm{min}$ and rocuronium $50 \mathrm{mg}$ was administered to facilitate tracheal intubation. Anesthesia was maintained by continuous infusion of remifentanil and desflurane. After administration of rocuronium, her entire body began to flush. Even though tracheal intubation was uneventful, the anesthesiologist felt very high resistance during manual bag ventilation. Peak airway pressure increased to $37 \mathrm{~cm} \cdot \mathrm{H}_{2} \mathrm{O}$ with a tidal volume of approximately $170 \mathrm{ml}$. The value of end tidal carbon dioxide tension was approximately $17 \mathrm{mmHg}$. Her systolic blood pressure decreased to $40 \mathrm{mmHg}$, and heart rate increased to $170 \mathrm{bpm}$. Because anaphylactic reaction was suspected, hydrocortisone $300 \mathrm{mg}$ was administered intravenously, and epinephrine $0.2 \mathrm{mg}$ was administered intramuscularly. Starting at about five minutes after administration of epinephrine, the peak airway pressure gradually decreased to $20 \mathrm{~cm} \cdot \mathrm{H}_{2} \mathrm{O}$, and skin flushing seemed to decrease. Her blood pressure and heart rate stabilized to $100 \mathrm{mmHg}$ of systolic blood pressure and $90 \mathrm{bpm}$ of heart rate. Surgery was cancelled. Thirty minutes after recovery from shock state, the train-of-four ratio by neuromuscular monitoring returned to over $90 \%$, and the patient regained consciousness. Her trachea was extubated. We did not measure the serum tryptase level because its measurement is not available at our hospital.

2.1. Skin Testing. A dermatologist was consulted and skin testing including the SPT and IDT for rocuronium, vecuronium, propofol, mepivacaine, and midazolam was scheduled. The skin prick test using administration of histamine as a positive control and saline as a negative control was undertaken in the operating room by the dermatologist. The concentrations of the test drugs used in the SPT and IDT are shown in Table 1. All drugs diluted to $1: 100$ in the SPT resulted in no reaction. However, among undiluted agents in the SPT, only rocuronium presented enlargement of flare and wheal, which indicated a positive allergic reaction. Other drugs did not present a positive reaction. The next day, the intradermal skin test was performed to identify whether cross-reaction between rocuronium and vecuronium could be observed. Only vecuronium was examined. Vecuronium, diluted at 1: $1000,1: 100$, and $1: 10$, was tested at 20 minute intervals, and no positive reaction was found at any dilution.

Laparoscopic ovarian cystectomy was rescheduled after one month. General anesthesia using vecuronium was safely performed. The surgery was uneventful, and the patient is doing well.

\section{Discussion}

Our patient developed circulatory shock, tachycardia, and bronchospasm with skin flush after induction of general anesthesia, which were suspected to be due to anaphylaxis. In the present case, the severity of anaphylaxis was grade III according to the scale developed by the Societe Francaise d' Anesthesie et de Reanimation (SFAR) [2]. The patient's
TABLE 1: Concentrations of agents in the skin prick test and intradermal test.

\begin{tabular}{lccccc}
\hline Substance & \multicolumn{2}{c}{$\begin{array}{c}\text { Skin prick test } \\
(\mathrm{mg} / \mathrm{ml})\end{array}$} & \multicolumn{3}{c}{ Intradermal test $(\mathrm{mg} / \mathrm{ml})$} \\
\hline Dilution & $1: 100$ & Undiluted & $1: 1000$ & $1: 100$ & $1: 10$ \\
Propofol & 0.1 & 10 & 0.01 & 0.1 & 1 \\
Midazolam & 0.05 & 5 & 0.005 & 0.05 & 0.5 \\
Mepivacaine & 0.1 & 10 & 0.01 & 0.1 & 1 \\
Rocuronium & 0.1 & 10 & 0.01 & 0.1 & 1 \\
Vecuronium & 0.1 & 1 & 0.01 & 0.1 & 1 \\
\hline
\end{tabular}

symptoms were severe, but she recovered by administration of intramuscular epinephrine.

When our patient developed these symptoms, we cancelled the surgical procedure. However, a study on a large series of patients who developed anaphylaxis during anesthesia suggested that there is no difference in anaphylaxisrelated outcome between patients in whom the surgical procedure was continued, and patients in whom the procedure was cancelled among cases where immediate recovery from anaphylaxis was obtained [8]. If a patient recovers from shock state, it may be safe to proceed with the procedure.

In the present case, mepivacaine, propofol, remifentanil, rocuronium, and povidone iodine used for disinfection were thought to be among the causative agents because the patient's condition was stable until the induction of general anesthesia. The causative drug was thought to be the drug used for induction of general anesthesia. In the present case, we performed SPT and IDT to identify the causative drug and also a safe drug to use for subsequent anesthesia. In the present case, undiluted rocuronium induced a flare and wheal which is a positive sign in the SPT. In the study of Dhonneur et al. [9], anesthesia-naïve volunteers received SPTs with various concentrations of rocuronium and vecuronium, and the authors found that $50 \%$ of the subjects had a positive skin reaction to undiluted rocuronium. And, in intradermal skin testing, non-mast-cell-mediated positive reaction are frequent with rocuronium and cisatracurium [10]. This irritating concentration of drug may induce a false-positive reaction. However, in patients with a history of hypotension and bronchospasm during anesthesia, the positive predictive value of the SPT was $98 \%$ [6]. In the same series by Berg et al. investigating cisatracum and rocuronium, no false-positive reaction was observed by undiluted agents in SPT [10]. We performed IDT using $1: 100$ and $1: 10$ dilution of vecuronium according to the guideline by SFAR. The dilution of $1: 10$ in vecuronium was the upper limit of testing concentration. Meters et al. demonstrated that, in healthy volunteers, reactive(false-positive) dilution in vecuronium was $1: 3.1$ and nonreactive(false-negative) dilution was $1: 31$. In the concentration, we have used in our patient, there is still small possibility of false-negative results in IDT. The efficacy of in vitro tests such as identification of the specific IgE against NMBA and BAT was proposed in recent studies; however, the negative predictive value is higher in skin testing compared with in vitro testing [11-13]. Leysen et al. suggested that quantification of specific IgE 
antibodies may be valuable in cases where negative skin testing is obtained in patients with a history of NMBAinduced anaphylaxis $[6,7]$.

In a recent animal study, it was shown that NMBA activates mast cell degranulation through Mas-related G-protein-coupled receptor member X2 (MRGPRX2) activation independent of the presence of $\operatorname{IgE}$ antibodies $[14,15]$. Activation of this receptor induces a non-IgEmediated response in NMBA-naïve patients such as a pseudo-allergic reaction. One important note is that in patients possessing this receptor, skin testing can give a falsepositive result even though patients do not have IgE antibody. This phenomenon induces relatively mild and transient symptoms in a dose-dependent manner and resembles an anaphylactoid reaction. In the present case, the patient had skin contact allergy to metal. The high probability of the presence of MRGPRX2 in keratinocytes in patients with urticaria indicates that there remains a possibility of pseudoallergic reaction in the present case.

The most remarkable point in this case is that, when a positive result in rocuronium and negative result in vecuronium were found in the SPT, it was worthwhile to proceed with the IDT of vecuronium to confirm the absence of cross-reaction. In the present case, subsequent general anesthesia using vecuronium was uneventful. Fisher et al. [16] reported three patients who developed subsequent allergic reaction to a NMBA that had negative responses on skin testing and IgE testing. They reported cross-reaction between decamethonium and succinylcholine, between pancuronium and alcuronium, and between rocuronium and vecuronium. Chiriac et al. [3] demonstrated that among 92 patients who presented hyperreaction to NMBA, 25 patients received an NMBA in subsequent general anesthesia, and 2 patients developed anaphylaxis with re-exposure to a negative skin-tested NMBA.

The usefulness of the skin prick test in identifying the drug that causes anaphylaxis should be re-evaluated. In patients who develop anaphylaxis during anesthesia, it is important to perform the SPT followed by the IDT not only to identify the causative drug but also to confirm which drug is safe to use in the subsequent anesthetic procedure.

\section{Conflicts of Interest}

The authors declare that they have no conflicts of interest.

\section{Acknowledgments}

The authors thank N.A.I corp. for preparing this manuscript. They also thank Professor Shinichi Anzai and Department of Dermatology, Musashikosugi Hospital Nippon Medical School, for evaluation of skin testing in this patient.

\section{References}

[1] D. G. Ebo, M. M. Fisher, M. M. Hagendorens, C. H. Bridts, and W. J. Stevens, "Anaphylaxis during anaesthesia: diagnostic approach," Allergy, vol. 62, no. 5, pp. 471-487, 2007.

[2] P. M. Mertes, F. Alla, P. Tréchot, Y. Auroy, and E. Jougla, "Anaphylaxis during anesthesia in France: an 8-year national survey," Journal of Allergy and Clinical Immunology, vol. 128, no. 2, pp. 366-373, 2011.

[3] A. M. Chiriac, C. Tacquard, N. B. Fadhel et al., "Safety of subsequent general anaesthesia in patients allergic to neuromuscular blocking agents: value of allergy skin testing," British Journal of Anaesthesia, vol. 120, no. 6, pp. 1437-1440, 2018.

[4] T. Heier and A. B. Guttormsen, "Anaphylactic reactions during induction of anaesthesia using rocuronium for muscle relaxation: a report including 3 cases," Acta Anaesthesiologica Scandinavica, vol. 44, no. 7, pp. 775-781, 2000.

[5] J. I. Reddy, P. J. Cooke, J. M. van Schalkwyk, J. A. Hannam, P. Fitzharris, and S. J. Mitchell, "Anaphylaxis is more common with rocuronium and succinylcholine than with atracurium," Anesthesiology, vol. 122, no. 1, pp. 39-45, 2015.

[6] J. Leysen, C. H. Bridts, L. S. De Clerck et al., "Allergy to rocuronium: from clinical suspicion to correct diagnosis," Allergy, vol. 66, no. 8, pp. 1014-1019, 2011.

[7] J. Leysen, A. Uyttebroek, V. Sabato, C. H. Bridts, L. S. De Clerck, and D. G. Ebo, "Predictive value of allergy tests for neuromuscular blocking agents: tackling an unmet need," Clinical \& Experimental Allergy, vol. 44, no. 8, pp. 1069-1075, 2014.

[8] P. H. M. Sadleir, R. C. Clarke, B. Bozic, and P. R. Platt, "Consequences of proceeding with surgery after resuscitation from intra-operative anaphylaxis," Anaesthesia, vol. 73, no. 1, pp. 32-39, 2018.

[9] G. Dhonneur, X. Combes, D. Chassard, and J. C. Merle, "Skin sensitivity to rocuronium and vecuronium: a randomized controlled prick-testing study in healthy volunteers," Anesthesia \& Analgesia, vol. 98, pp. 986-989, 2004.

[10] C. M. Berg, T. Heier, V. Wilhelmsen, and E. Florvaag, "Rocuronium and cisatracurium-positive skin tests in nonallergic volunteers: determination of drug concentration thresholds using a dilution titration technique," Acta Anaesthesiologica Scandinavica, vol. 47, no. 5, pp. 576-582, 2003.

[11] A. Trautmann, C. Seidl, J. Stoevesandt, and C. S. Seitz, "General anaesthesia-induced anaphylaxis: impact of allergy testing on subsequent anaesthesia," Clinical \& Experimental Allergy, vol. 46, no. 1, pp. 125-132, 2015.

[12] L. F. Ramirez, A. Pereira, A. M. Chiriac, M.-C. Bonnet-Boyer, and P. Demoly, "Negative predictive value of skin tests to neuromuscular blocking agents," Allergy, vol. 67, no. 3, pp. 439-441, 2012.

[13] J. Li, O. G. Best, M. A. Rose et al., "Integrating basophil activation tests into evaluation of perioperative anaphylaxis to neuromuscular blocking agents," British Journal of Anaesthesia, vol. 123, no. 1, pp. e135-e143, 2019.

[14] B. D. McNeil, P. Pundir, S. Meeker et al., "Identification of a mast-cell-specific receptor crucial for pseudo-allergic drug reactions," Nature, vol. 519, no. 7542, pp. 237-241, 2015.

[15] G. Porebski, K. Kwiecien, M. Pawica, and M. Kwitniewski, "Masreated G protein-coupled receptor-X2 (MRGPRX2) in drug hypersensitivity reactions," Frontiers in Immunology, vol. 20, 2018.

[16] M. M. Fisher, D. Merefield, and B. Baldo, "Failure to prevent an anaphylactic reaction to a second neuromuscular blocking drug during anaesthesia," British Journal of Anaesthesia, vol. 82, no. 5, pp. 770-773, 1999. 